\title{
Magnetoresistive (MR) Sensors for Angle-, Path- and Current Measurement in Harsh Environments
}

\author{
Dr. R. Slatter ${ }^{1}$ \\ 1 Sensitec GmbH, Lahnau, Germany \\ rolf.slatter@sensitec.com
}

\begin{abstract}
:
Nowadays, magnetoresistive (MR) sensors are used in a wide range of applications. In general, the MR-effect describes the change of the electrical resistance in an external magnetic field. MR sensors are not only used for measuring magnetic fields and rotational or linear motion, but also for noncontact switching applications and furthermore for highly dynamic current measurement. This is largely the result of increasingly complex demands on the sensors for e.g. high performance electrical drives. The sensors must not only be accurate and dynamic, but must also be robust under difficult operating conditions and exhibit very high reliability. Due to their physical working principle and their small size, MR sensors are especially suited to work in harsh environments like high or low temperature, radiation, pressure or mechanical shock.

This paper describes the principle of operation and benefits of MR sensors. This will be followed by a description of practical application examples from the automotive, oil \& gas, renewable energy and space fields, where MR sensors are successfully applied in very small envelopes at very low /very high temperatures, under high pressure, high mechanical loading and under strong radiation.
\end{abstract}

Key words: Magnetoresistive, MR, magnetic sensor, harsh, temperature, shock, radiation, pressure

\section{Introduction}

The magnetoresistive effect is probably best known from the read heads of computer hard discs or from magnetic memory (MRAM) applications, but it is also well suited to uses in sensor technology. It has a long history, the anisotropic magnetoresistive (AMR) effect being first discovered in 1857 by Lord Kelvin. The AMR effect occurs in ferromagnetic materials, such as nickel-iron layers structured as strip elements, whose specific impedance changes with the direction of an applied magnetic field. Due to a special structure of the strips the resistance change is proportional to the applied magnetic field over a wide range.

However, the MR-effect did not experience widespread use until the early 1980s, when the first MR-based read heads were implemented in hard disc drives. The first industrial applications for MR-based sensors followed at the beginning of the 1990s, since when the number of applications has increased dramatically. The applications are not only limited to terrestrial use - MR sensors are used to control the electric drives used on "Curiosity", the Planetary Rover that landed successfully on Mars in August 2012. MR sensors are also used extensively in safety-critical automotive applications, for example in wheel speed sensors for the ABS (anti-lock braking) system or in steering angle sensors for the ESP (electronic stability program) system.

The magnetoresistive effect is particularly attractive for the use in harsh environments. The small envelope of the sensors and the robust working principle make them suitable for the use in high and low temperature applications, in places with high radiation e.g. on space missions, under high pressure and in applications where the sensor system is exposed to high mechanical shock or high vibration levels.

\section{Magneto-Resistive Sensors \\ Magnetoresistive (MR) Basics}

Magnetoresistive (MR) sensors are firmly established in automobiles, mobile telephones, medical devices, wind turbines, machine tools or industrial robots: be it for the measurement of path, angle or electrical current, or as an electronic compass. Originally developed for data storage applications, the different MR effects open up new measurement possibilities for sensors, not only in terrestrial applications, but also in space applications. 
MR sensors are robust, reliable, precise and miniaturized. This combination of features is leading to continuous growth in the application field of MR sensors. The extremely low power consumption of MR sensors makes them ideal for wireless, autonomous sensor applications. They present the developers of many different types of mechanism or instrument with completely new possibilities to measure angle, path, electrical currents or magnetic fields.

The magnetoresistive effect has been known for more than 150 years. The British physicist William Thomson, later known as Lord Kelvin, discovered that the electrical resistance of a conductor changed under the influence of a magnetic field. However, this effect could first be used industrially more than 120 years later, during the late 1970s, in combination with thinfilm technologies derived from the semiconductor industry. The intelligent arrangement of thin-film structures within a sensor enabled the development of many different sensor types for measuring the angle, strength or gradient of a magnetic field. The effect discovered by Thomson was named the "anisotropic magnetoresistive effect" (AMR) and resulted in a resistance change of just a few percent. Nevertheless this effect was used million-fold in the production of read-heads for hard discs. At the end of the 1980s the "giant magnetoresistive effect" (GMR) was discovered independently by Prof. Grünberg at the Forschungszentrum Jülich in Germany and by Prof. Fert at the University of Paris in France. Here the resistance change was more than $50 \%$, which opened up even more applications for MR sensors. This discovery was awarded the Nobel Prize for Physics in 2007.
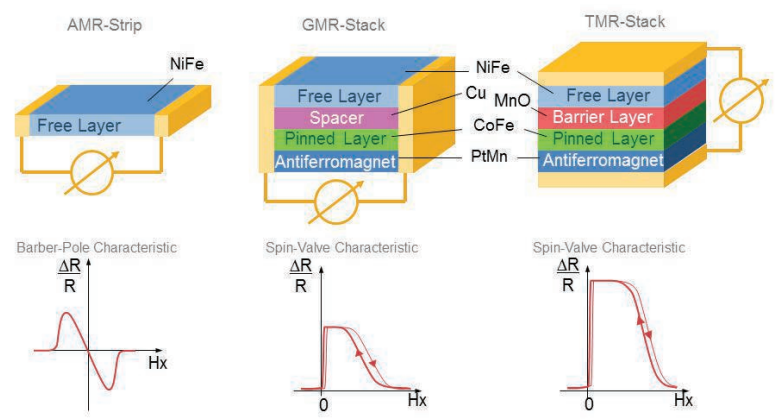

Fig. 1: Comparison of MR technologies

In the meantime the read-heads of hard discs are almost exclusively based on the "tunnel magnetoresistive effect" (TMR), which can exhibit a resistance change of several hundred percent under laboratory conditions. This technology has additional features that are not only interesting for storage technology, but also for sensors. For example, TMR-based sensors have an extremely high sensitivity and a very low power consumption.

Fig.1 shows a schematic comparison of the different MR layer structures and Tab.1 compares their basic characteristics for commercially available products. For sensor applications the AMR, GMR and TMR effects are complementary, each offering specific advantages that can be of benefit in different applications [1][2].

Tab. 1: Differences between MR technologies

\begin{tabular}{|c|c|c|c|}
\hline & AMR & $\begin{array}{c}\text { GMR } \\
\text { (Spin- } \\
\text { Valve) }\end{array}$ & TMR \\
\hline $\begin{array}{c}\text { Signal } \\
\text { strength } \\
(\Delta \mathrm{R} / \mathrm{R})\end{array}$ & $<4 \%$ & $<15 \%$ & $<200 \%$ \\
\hline $\begin{array}{c}\text { Direction } \\
\text { of } \\
\text { current } \\
\text { flow }\end{array}$ & $\begin{array}{c}\text { Parallel } \\
\text { to layer } \\
\text { plane }\end{array}$ & $\begin{array}{c}\text { Parallel } \\
\text { to layer } \\
\text { plane }\end{array}$ & $\begin{array}{l}\text { Perpendicular } \\
\text { to layer plane }\end{array}$ \\
\hline Topology & Meander & Meander & Point \\
\hline
\end{tabular}

\section{MR sensor applications}

MR sensor chips can be designed to form angle sensors, field sensors, position sensors and current sensors. Most of these use a Wheatstone bridge configuration with e.g. stripes of MR material forming the resistors.

Current sensors based on the MR effect use the magnetic field generated by a live conductor. MR field sensors measure strength and / or direction of a magnetic field, e.g. the earth magnetic field for compass applications. For angle or position measurements using MR sensors, a magnet or a magnetic scale has to be used to generate a varying magnetic field. A standard configuration is the use of a permanent magnet at the end of a motor shaft to measure its angular position. Magnetic scales can be either active (magnetic north and south poles "written" onto a magnetic material) or passive (toothed wheel or toothed bar; sensor detects distortion of magnetic field caused by the teeth).

MR sensors are used in applications from the automotive (e.g. steering angle, wheel speed, electric motor commutation) as well as the consumer (e.g. smartphones, gaming consoles) and the industrial sector (e.g. industrial automation, drive systems, renewable energies, 
space- and aircrafts, (bio)-medical devices, etc.).

\section{MR Sensors in harsh environments MR sensors and harsh environments}

MR sensors are suited for high $\left(\sim 200{ }^{\circ} \mathrm{C}\right)$ and low (no limit) temperature applications. Only at temperatures far above $200{ }^{\circ} \mathrm{C}$ will the properties of the MR layers be degraded or destroyed. Due to their small size and thus weight, mechanical shock has no significant impact on MR sensors. Only very high radiation can affect the MR effect.

\section{Radiation}

Magnetoresistive sensors are particularly interesting for space applications, because small mass, small volume, high robustness under difficult operating conditions or low power consumption, are especially important here. The Mars Rovers "Spirit", "Opportunity" and "Curiosity" all feature numerous electrical actuators, most of which are controlled using MR-based magnetic encoders "made in Germany". The experience of Mars applications has provided the basis for a new generation of MR sensors for space and aircraft applications.

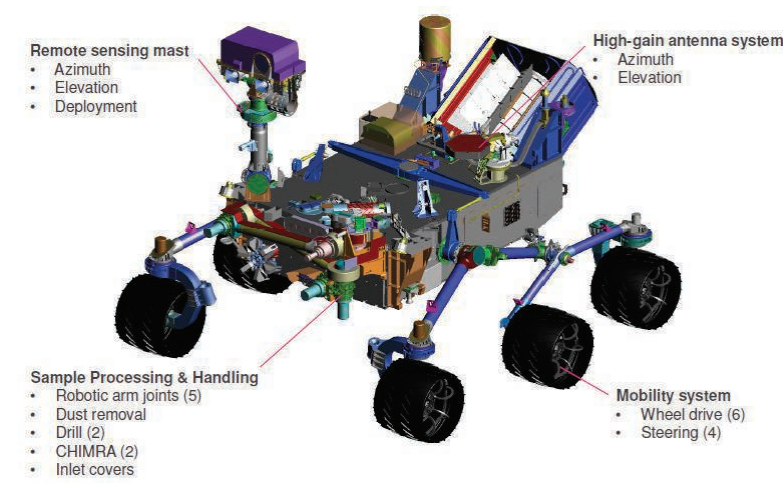

Fig. 2: Sensitec AMR sensors used in "Curiosity" (source: NASA/JPL-Caltech)

Radiation is a huge problem for semiconductor based sensors (e.g. Hall effect based magnetic sensors) and can cause severe malfunction. The broad temperature range and the mechanical load as well as the high risk of contamination during operation complicate the use of optical sensors. These limitations do not apply to MR sensors.

The environmental conditions during transportation to and operation on Mars and the resulting requirements for the sensors are quite challenging:

- Wide temperature range: $-130{ }^{\circ} \mathrm{C}$ to $+85{ }^{\circ} \mathrm{C}$

- Solar and cosmic radiation
- Mechanical shock (20g during rocket launch, $8 \mathrm{~g}$ during operation on Mars)

- Small and light weight

- Low power consumption

- High reliability

Sensitec MR sensors have been used in space applications for more than 12 years: 39 magnetoresistive Sensors made by Sensitec were used in each of the two rovers ("Spirit" and "Opportunity") of the "Mars Exploration Rover Mission" (MER). The two rovers were launched in 2003 and contained Sensitec AMR sensors as part of the magnetic motor encoder system integrated into special motors fabricated by the Swiss company Maxon Motor AG. Until now, none of the MR sensors used in "Spirit" and "Opportunity" have failed.

At the end of 2011 another Mars-Mission "Mars Science Laboratory" (MSL) started. The rover "Curiosity" landed on August 6", 2012 on Mars. Figure 2 shows the rover and depicts the application of all $40 \mathrm{MR}$ sensors used in "Curiosity".

\section{High Temperature}

There are several examples for MR sensors in high temperature applications. One of the high volume applications is a wheel speed sensor based on AMR sensor chips. A very important requirement is the extremely high reliability $(<$ $0.1 \mathrm{ppm}$ field return rate) despite e.g. high operating temperatures of up to $195{ }^{\circ} \mathrm{C}$. The complete sensor assembly and the injection molded MR sensor device are depicted in Figure 3. More than 120.000.000 Sensitec AMR sensor chips have been delivered for this application without a single field return caused by the MR technology.

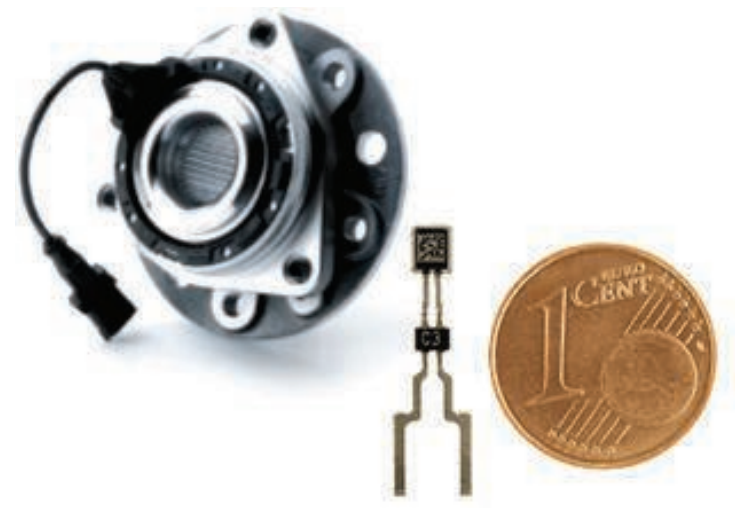

Fig. 3: AMR based wheel speed sensor for automotive applications (source: Continental AG)

Another example for high operating temperatures in combination with high pressure 
is the use of MR sensors in calipers (open hole tools) for borehole diameter measurement. Antares Datensysteme $\mathrm{GmbH}$ uses Sensitec AMR sensors in 4-arm calipers. Since 2005 several thousand systems have been fabricated; the field-return rate is extremely low. The 4-arm caliper measurements provide diameter data of the borehole in two directions. The measurements are used to

- describe the shape of the borehole, in particular washout and restrictions in 2 directions

- provide information on the build-up of mudcake

- calculate borehole and cement volumes (in respect to casing size)

- correct parameters of other logs.

The caliper is specified for operation up to 175 ${ }^{\circ} \mathrm{C}$ (350 ${ }^{\circ} \mathrm{F}$ ) and $140 \mathrm{MPa}$ (20.000 psi); the sensor system is specified for temperatures up to $205{ }^{\circ} \mathrm{C}$. Figure 4 shows a schematic of the 4arm caliper. A detailed view of the sensor seat can be seen in fig. 5 .

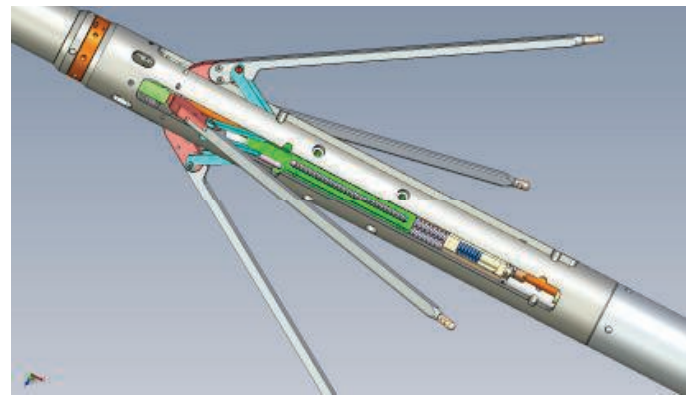

Fig. 4: Schematic of the Antares 4-arm caliper (source: ANTARES Datensysteme $\mathrm{GmbH}$ )

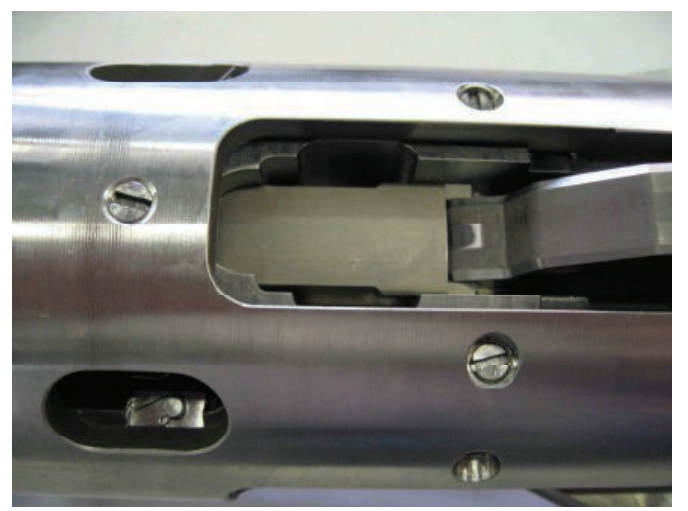

Fig. 5: Detail of the sensor position in the Antares 4arm caliper (source: ANTARES Datensysteme $\mathrm{GmbH})$

GMR sensors can also be used in high temperature applications: A valve lift sensor developed by Sensitec can measure the valve movement (lift and rotation) in a fired engine (environmental temperature approx. $150{ }^{\circ} \mathrm{C}$ ). Currently these sensors are used in motor test- stands. The requirements on the sensor performance are quite challenging: a contactless measurement principle with a resolution below $10 \mu \mathrm{m}$ suited for the measurement of linear speed above $100 \mathrm{~m} / \mathrm{s}$. All of these requirements can be met with a GLM Tooth Sensor Module which detects the deformation of the magnetic field caused by a tooth structure in the valve (see Fig. 6 and 7). The measurement is not even affected by oil contamination.

The VLS sensors are used in the development phase of gasoline engines to measure dynamic effects and to detect errors in the valve train. In the very near future these sensors will not only be applied to test-stands but also to racing cars to improve the engine control and thus the performance of the car.

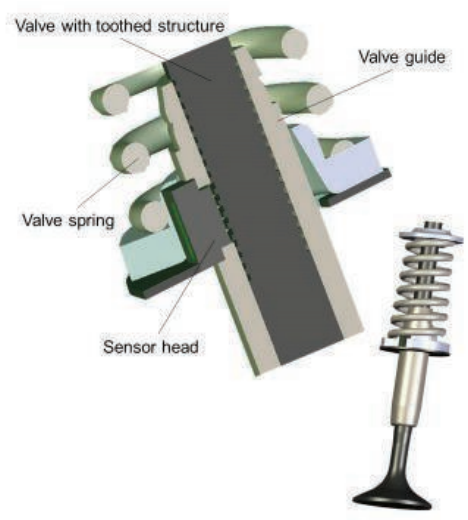

Fig. 6: Operating principle of VLS sensor

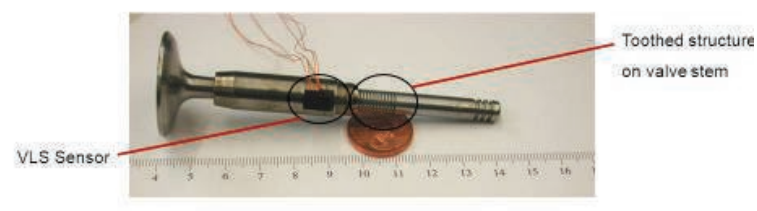

a) Integration of the VLS sensor into the valve guide

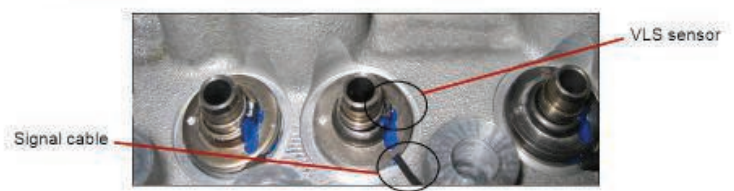

b) Assembly of the modified valve guide into the cylinder head

Fig. 7: Valve Lift Sensor for IC-Engine Testing

\section{Low Temperature}

One of the low temperature applications MR sensors are used in has already been discussed earlier. The temperature requirements for motor encoders used in the Mars missions are specified as -130 to $+85{ }^{\circ} \mathrm{C}$.

Figure 8 shows a GMR base valve lift sensor from above dipped into liquid nitrogen during 
qualification. The sensor was not harmed by the treatment and remained fully functional.

Another example for low temperature environments are current sensors for MoreElectric Aircraft applications. Following on from the POA (Power Optimized Aircraft) and More Open Electrical Technologies (MOET) initiatives, the European Union is now partfunding the Cleansky Initiative, where one of the main activities is the replacement or enhancement of hydraulic and pneumatic systems by means of electrically driven actuators, supported by the latest sensor technology. The focus is on the electrification of high-lift systems, the landing gear and the environmental control system. MR sensors have been applied successfully in demonstrators in all three areas for either angle sensing or current sensing applications [3]. In this paper the application of highly integrated current sensors in an electrical environmental control system (E-ECS) will be described.

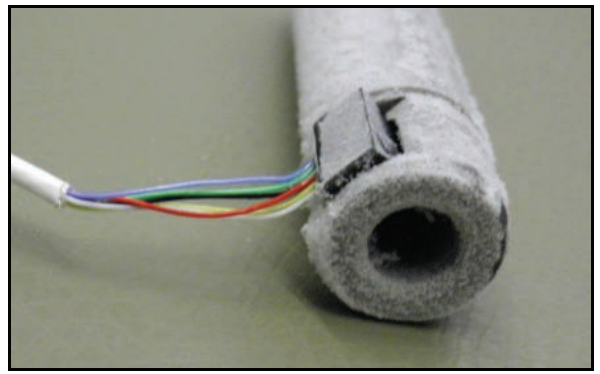

Fig. 8: Valve lift sensor dipped into liquid nitrogen without influence on functionality

At aircraft level, the electrification of the ECS is a key enabler of the More Electric Aircraft, as it is the biggest steady state power consumer during aircraft cruise. An electrical ECS could also enable the saving of a lot of fuel, due to its consequential adaptability and a reduced impact on the operation of the engine [4],[5]. The task of an Environmental Control System (ECS) is to control air temperature, to pressurize relevant aircraft compartments, to provide sufficient ventilation and fresh air to passengers, to control the level of humidity within acceptable limits in the cabin, and to remove pollutants. A conventional ECS uses air bled from the engine compressor to provide conditioned air to the cabin. The numerous benefits of an electrical ECS include improved engine design, better fuel efficiency and improved functionality, because the E-ECS can be operated independently from the operational cycles of the engine [6].

Within the Cleansky project a German aerospace supplier has the task of optimizing the E-ECS. The target is to increase the power density of the power electronics, compared to previous solutions, by a factor of 4 . CFS1000 current sensors (see Figure 9) which use Sensitec AMR sensors [7] have been selected for the following reasons:

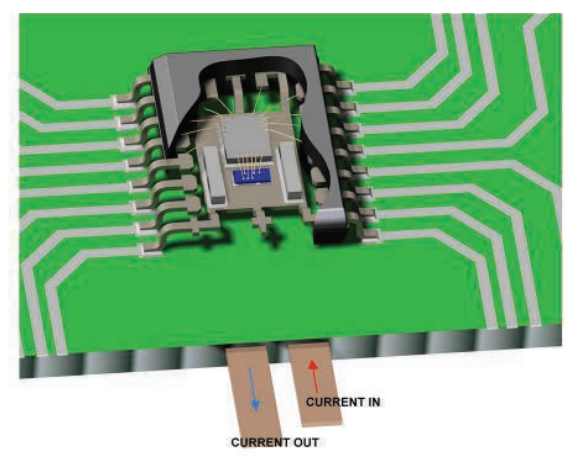

Fig. 9: U-shaped conductor embedded in $P C B$ underneath CFS1000 current sensor

- $\quad$ Extremely small size to reduce volume and weight of the complete power electronics

- High shock and vibration resistance of the automotive-qualified product

- High accuracy to allow more accurate dimensioning (and therefore downsizing) of other electronic components

The AMR current sensors are used to measure rated current of up to $65 \mathrm{~A}$ passing through a conductor embedded in the power electronics PCB (Fig. 9). The current sensors are mounted between the IGBT power pack and the threephase motor (see Fig. 10). The application of MR-based current sensors has enabled an extremely compact design of the power electronics for the E-ECS [8].

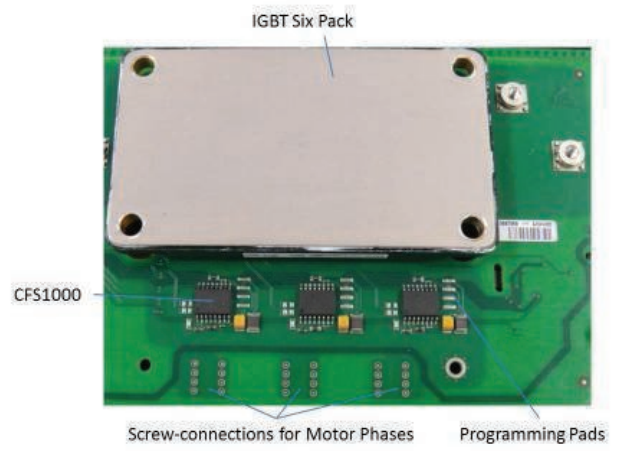

Fig. 10: PCB from Electrical Environmental Control System (Source: Liebherr Elektronik $\mathrm{GmbH}$ )

\section{Mechanical Shock}

A good example for MR sensors under high mechanical shock is the use of GMR Tooth Sensors for process control in a Böllhoff riveting tool. The tool is depicted in Figure 11. The drive unit is equipped with a re-filled tooth structure as a passive magnetic scale - an active scale will lose its magnetization during operation due 
to the high shock. Two Sensitec GLM modules sample this scale and provide the position measurement information.

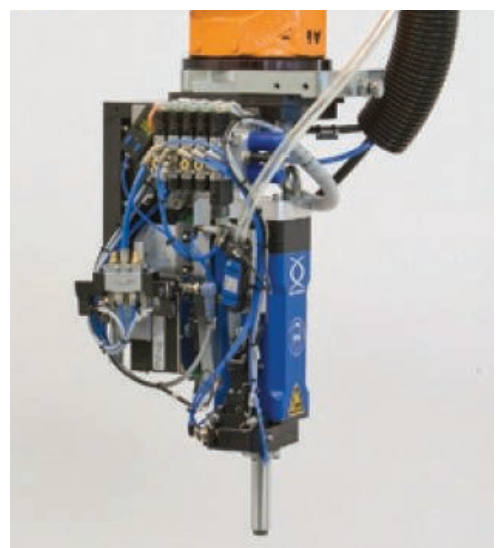

Fig. 11: Riveting tool (source: Wilhelm Böllhoff $\mathrm{GmbH)}$

Mechanical shock on the sensor unit up to $1500 \mathrm{~g}$ has been measured during operation with resulting forces above $10 \mathrm{kN}$. 300.000 riveting sequences under maximum load are guaranteed, but typically more than 500.000 sequences can be done without failure. Figure 12 shows a typical path-time diagram recorded with the MR sensor system during riveting.

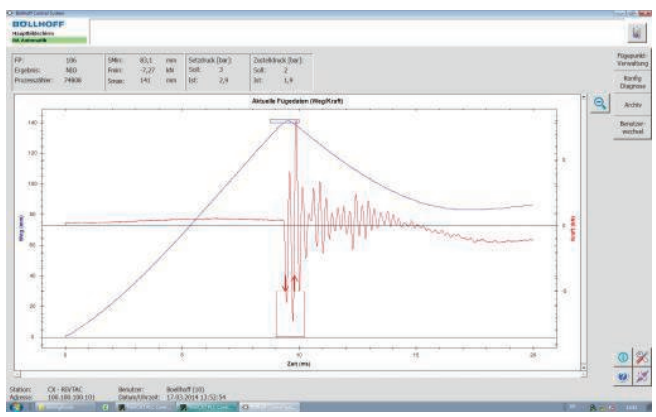

Fig. 12: Path-time diagram during recorded during riveting (source: Wilhelm Böllhoff $\mathrm{GmbH}$ )

\section{Additional Advantages of MR Sensors in Harsh Environments}

What is common to all MR effects is the low power consumption, which is particularly important in the field of wireless sensor applications. Autonomous, wireless sensors are particularly important in difficult or poorly accessible operating environments. Due to the high power typically drawn by the wireless communications electronics it is essential that the sensor itself is as energy efficient as possible. Until recently, the high power consumption of the sensor and the signal conditioning electronics have resulted in a battery life that is too short for many practical industrial applications. This means that outside the consumer market wireless sensors are only currently used in niche applications.
Magnetoresistive sensor technology has the potential to help change this situation [9].

\section{Summary}

An increasing amount of applications for angular, position or current measurement in harsh or demanding environments requires the development of sensors and sensor systems that can withstand the rough conditions in these applications.

Magnetic sensors based on the MR effect offer significant benefits compared to sensors based on other physical effects, such as optical, inductive or capacitive sensors. MR sensors have already proven their excellent ability for operating in high and low temperature applications, as well as in high radiation and high pressure environments and under high mechanical impact (shock). MR sensors open up new opportunities for design engineers to measure mechanical and electrical values in locations that previously were not possible, either due to the limited available space or the harsh operating environment.

\section{References}

[1] Doms, M. et al; "AMR vs. GMR vs. TMR Eigenschaften, Unterschiede, Anwendungen", Proc. of 5. Mikrosystemkongress, Aachen, 2013

[2] Slatter, R.; "Magnetische Mikrosysteme in Industrie-, Automobil- und

Raumfahrtanwendungen", Proc. of 4.

Mikrosystemtechnikkongress, Darmstadt, 2011

[3] Schäfer, I., "Aviation Sensor Requirements: Do they fit to MR Technology?", Proc. of 11th MR Symposium, Wetzlar, 2011

[4] Faleiro, L. et al, "Integrated Equipment Systems for a More Electric Aircraft - Hydraulics and Pneumatics", Proc. of 24th International Congress of the Aeronautical Sciences, 2004

[5] AbdElhafez, A.A., Forsyth, A.J., „A Rreview of More Electric Aircraft“, Proc. of 13th International Conference on Aerospace Science and Aviation Technology, Cairo, 2009

[6] Galzin, G. et al, "Electrical Environmental Control System", Proc. of More Electric Aircraft Forum, 2009

[7] Scherner, S. et al, "Integrated Current Sensor based on Magnetoresistive (MR) Technology, Proc. of PCIM Conference, Nuremberg, 2012

[8] Slatter, R., Scherner, S., "Highly integrated magnetoresistive current sensors for aerospace applications", Proc. AMA Conference Sensor, Nuremburg, 2013

[9] Slatter, R., Doms, M., "Energy efficient magnetoresistive sensors for low-power and wireless applications", Proc. of SSI Conference, Vienna, 2014 